Institute for Endocrine Sciences, Shahid Beheshti University of Medical Sciences, Tehran, Iran

Introduction Attributable Fraction is the commonest method of describing the proportion of a health outcome attributable to an exposure in an exposed group. It applies to binary variables. Many variables are continuous; changing them to binary variables results in loss of information. Using traditional analyses we compared the importance of cardiovascular disease (CVD) risk factors in a continuous form.

Methods A population based cohort study including 1802 men and 2301 women aged $\geq 40$ years in Tehran. We considered modifiable continuous exposures at baseline and CVD events during 8.5 years of follow-up. Using factor analysis we extracted some uncorrelated and standardised factors, each related to a cluster of continuous variables with the same general feature (eg, systolic and diastolic blood pressure as blood pressure factors or body mass index and waist circumference as anthropometric factor); then, a Cox regression including these factors as scores was conducted to estimate the $\mathrm{RR}$ of the last quintile to the first for each factor. Finally we compared these similar RRs in the model using the Wald test.

Results Anthropometric, blood glucose, blood pressure and cholesterol factors were extracted. The total variance explained by factors was $88.6 \%$ in men and $87.3 \%$ in women. In men all factors had the nearly the same RRs ranging from 1.7 to 2.2 but in women the RR of cholesterol was significantly higher than the others (3.4 vs $1.7-2.5$ ).

Conclusion To prevent CVD, all clusters of risk factors should be considered in control programs. Hypercholesterolaemia maybe more important in women.

\section{SP1-82 COLORECTAL CANCER MORTALITY AND ITS POSSIBLE RELATIONSHIP WITH EXPOSURE TO INDUSTRIAL POLLUTION IN SPAIN}

doi:10.1136/jech.2011.142976n.59

\begin{abstract}
${ }^{1,2} \mathrm{G}$ Lopez-Abente, ${ }^{* 1,2} \mathrm{~J}$ García-Pérez, ${ }^{1,2} \mathrm{P}$ Fernández-Navarro, ${ }^{1,2} \mathrm{E}$ Boldo, ${ }^{1,2} \mathrm{R}$ Ramis. ${ }^{1}$ National Centre for Epidemiology, Carlos III Institute of Health, Madrid, Spain; ${ }^{2}$ CIBER Epidemiología y Salud Pública (CIBERESP), Madrid, Spain
\end{abstract}

The European Pollutant Release and Transfer Register (PRTR) constitute a valuable resource for monitoring health effects of industrial pollution. Our objective is to ascertain whether there might be excess colorectal cancer (CRC) mortality among populations residing in the vicinity of Spanish industrial installations governed by the PRTR.

Methods An ecological study of CRC mortality at a municipal level (8098 towns), over the period 1997-2006. We conducted an exploratory "near vs far" analysis to estimate the RRs of towns at a distance of $<2 \mathrm{~km}$ from installations. The analysis include 24 industrial groups. RRs and their $95 \%$ credible/CI were estimated using Poisson regression models, using two approaches: (A) a conditional autoregressive Bayesian model, with explanatory variables; and (B) a mixed regression model. Integrated nested Laplace approximations were used as a Bayesian inference tool.

Results Statistically significant RR were detected in the vicinity of metal production and processing (RR 1.07; 95\% CI 1.01 to 1.12), mining (RR 1.26; 95\% CI 1.08 to 1.46), paper, pulp and board manufacture (RR 1.07; 95\% CI 1.01 to 1.14), ceramic factories (RR $1.05 ; 95 \%$ CI 1.00 to 1.10 ) and food and beverage production (RR $1.07 ; 95 \%$ CI 1.03 to 1.11 ).

Conclusions Residing in the vicinity of PRTR-registered industries may constitute a risk factor for CRC, since a higher mortality was detected in both men and women residing in towns with such industries nearby. Some of the differences between men and women suggest that there may be a strong, little-studied component of occupational exposure. SP1-83 ALCOHOL CONSUMPTION AND URBANISATION IN NORTH
INDIA: A COMMUNITY SURVEY

doi:10.1136/jech.2011.142976n.60

V Gupta Ritvik, ${ }^{*}$ A Krishnan. All India Institute of Medical Sciences, Delhi, India

Introduction Indian is in a state of epidemiological transition with changes in urbanisation and risk factor profiles. In this context, our objective was to study the epidemiology of alcohol use among urban and rural populations.

Methods The study was conducted in Ballabgarh block, India, during January-October 2006 using the WHO-STEPs approach. Participants were selected using multi-stage sampling for rural and urban stratum (sub-divided across town and slum). We targeted enrolling 250 male and female participants each in 10 year agegroups across 25-65 years and enrolled 5005 participants. In a $20 \%$ sub-sample, information was collected about the exposure to health communication messages regarding alcohol.

Results One rural female reported current alcohol use. The prevalence of current consumption of alcohol, defined as consumption within preceding 12 months, was highest among urban males (26.2\%; 95\% CI 20.8 to 32.1) followed by urban slums $(25.6 \%$; $95 \%$ CI 19.2 to 32.4 ) and rural (23.2\%; 95\% CI 18.2 to 28.9). Among alcohol consumers $15.3 \%$ urban, $7.3 \%$ slum and $15.3 \%$ rural males reported reduction in alcohol use in preceding 1 year. $7.2 \%$ urban $10.9 \%$ slum and $7.2 \%$ rural respondents who were current alcohol users reported intermittent cessation of alcohol consumption in preceding 1 year. $59.2 \%(n=603)$ urban, $27.2 \%(n=185)$ urban-slum and $39.5 \%(n=646)$ rural respondents reported receiving information (IEC) on health effects of alcohol with Mass media as the most common source of information.

Conclusion Alcohol is a public health problem among males in Ballabgarh area. Interventions should focus on alcohol cessation services and improved IEC. Urbanisation does not appear to be associated with alcohol use in this community.

\section{SP1-84 DIFFERENT METHODS TO CALCULATE POPULATION ATTRIBUTABLE FRACTION OF RISK FACTORS FOR CARDIOVASCULAR DISEASES: TEHRAN LIPID AND GLUCOSE STUDY (TLGS)}

doi:10.1136/jech.2011.142976n.61

S S Azimi, ${ }^{*}$ D khalili, F Hadaegh, P Yavari. Prevention of Metabolic Disorders Research Center, Tehran, Iran

Background The population attributable fraction (PAF) that estimates potentially community-level effect of risk factors can be useful in planning public health interventions. This study compared different methods for calculating adjusted PAFs for cardiovascular diseases (CVD) in a cohort study with 10 years of follow-up.

Methods Baseline data were employed from 6630 participants (3746 women) above 30 years old and 558 CVD events (238 women) detected during follow-up. Unadjusted approach using Levin's formula, Miettinen formula approach using adjusted OR and HR estimated from logistic and Cox regression and direct estimation of average PAF from logistic regression using Rückinger method, were applied.

Result Estimated PAFs, using HR comparing OR, in both Levin's and Miettinen's formula, with tiny decrease, gave very similar results. However, according to the average PAF method, frankly, we reach to lower fractions; highest modifiable cardiovascular risk factor PAFs, in sequence, was hypertension $(16.2 \%)$, smoking $(14.8 \%)$, diabetes $(10.1 \%)$, hypercholesterolaemia (8.5\%) for men, and hypertension (25.6\%), diabetes (18\%), hypercholesterolaemia $(10.7 \%)$, for women. Also PAF of Age $\geq 60$ years and premature family history of CVD, as most important non-modifiable CVD risk 
factors, were $18.4 \%, 11.2 \%$ and $4.4 \%, 6.9 \%$ for men and women respectively.

Conclusion If individual data (eg, cohort and case-control studies) are accessible then the direct estimation of average PAF provides more realistic results. Besides, it seems that the order of important risk factors is the same in men and women except smoking.

\section{SP1-85 TROUBLED SLEEP: A CROSS-NATIONAL STUDY OF THE INFLUENCE OF AGE, HEALTH, SOCIAL AND PSYCHOSOCIAL FACTORS ON SLEEP}

doi:10.1136/jech.2011.142976n.62

\begin{abstract}
${ }^{1,2} \mathrm{G}$ Netuveli, ${ }^{1,2}{ }^{2}$ Webb, ${ }^{1,2} \mathrm{D}$ Blane. ${ }^{1}$ Imperial College London, London, UK; ${ }^{2}$ Inter-
\end{abstract} national Centre for Life Course Studies in Society and Health, UCL, London, UK

Introduction Trouble with sleep is often accepted as a part of ageing with its prevalence reported up to half of the population surveyed. However it has been persuasively argued that in healthy ageing individuals, sleep need not diminish significantly or be of poor quality and that it is the chronic diseases and functional limitations that produce sleeping problems in the elderly. The objective of this study is to compare the prevalence of sleep problems and assess the influence of age, health and psycho-social factors in a sample of European countries.

Method Data of non-institutionalised Europeans above 50 years from the Study of Health, Ageing and Retirement in Europe were used. The outcome was a binary variable based on self reported sleep problems. Explanatory variables included age, gender, and various health, social and psycho-social factors. Statistical analysis was done using multiple logistic regression and multi-level models.

Result The prevalence of sleep problems ranged from 18\% in Greece to $48 \%$ in Poland; Greece was an exception with other Southern European countries reporting more sleep problems. Age was significant factor only for women who had greater probability of sleep problems than men. Health was the major influence on sleep problems but psychosocial factors like trust in others and social factors like receiving help were also significantly associated.

Conclusions Health is the major influence on sleep with age significant only for women. However there are significant differences among European countries in the prevalence of sleep problems.

\section{SP1-86 FOLLOW-UP AND INCIDENT OUTCOMES IN A LONGITUDINAL STUDY IN BRAZIL (ELSA-BRASIL)}

doi:10.1136/jech.2011.142976n.63

S Barreto, R Ladeira, * V Passos, F Diniz, S Kelles. UFMG, Belo Horizonte/Minas Gerais, Brazil

Introduction Baseline data from Longitudinal Study of Adults Health (ELSA-Brasil), a cohort that investigates incidence and predictors of chronic diseases among 15000 civil servants aged 35-74 years from six universities in Brazil, was fully accomplished in December 2010. From now on, main challenges are: avoid loss to follow-up over time, correct identification, and classification of incident outcomes.

Methods Follow-up data collection relies on annual phone interview to identify hospitalisations and emergency department visits, 3-year examination in research centres, spontaneous participant report and linkage with available databases from human resources department of universities and death certificates. Events of interest include: acute myocardial infarction, unstable angina, heart failure, peripheral artery disease, myocardial revascularisation, resuscitated sudden death, stroke, transient ischaemic attack, incident diabetes, diabetes related complications, chronic kidney disease, cancer and all-causes mortality. Besides, intermediate outcomes such as hypertension, obesity, cognitive decline, kidney and endothelial dysfunction will be ascertained. To guarantee the uniformity of the assessment, an ELSA Outcome Committee performs a reiterated verification of the protocols, scripts and certifies periodically researchers which are in direct contact with the population under study. The Morbidity and Mortality Committee aggregates specialists from the six research centres, in order to perform events classification according to standardised protocols.

Conclusions The great number of exposures studied will allow investigate many associations with outcomes of interest. Besides that, a promising issue of this study is the biological samples and DNA bank of all participants which will also allow nested casecontrol analysis.

\section{SP1-87 PREVALENCE AND CHARACTERISTICS OF EARLY CHILDHOOD OBESITY IN TURKEY}

doi:10.1136/jech.2011.142976n.64

S Tezcan,* T Adali, E K Yigit. Hacettepe University, Ankara, Turkey

Introduction Developing societies experience a nutritional transition in line with their epidemiological transition. As a diet rich in carbohydrates and fats and poor in fibre spreads, one of the major consequences of the nutritional transition emerges: Obesity. Among all age groups, childhood obesity is especially important, since it is known that it is likely to lead to adulthood obesity; an important risk factor for cardiovascular disease and diabetes. This study focuses on early childhood (under age 5) obesity in Turkey. This emerging issue is gaining importance Turkey's public health agenda, and there are already local studies to estimate the prevalence of childhood obesity. However, no national estimates have been obtained until recently, and this study presents the findings obtained for the further analysis study of the Turkey Demographic and Health Survey, 2008.

Methods Data were obtained from the anthropometric measurements in this survey.

Results The national prevalence of childhood obesity was calculated as $5.8 \%$. It was higher in urban areas (6.7\%) than rural areas $(3.5 \%)$. It was also higher in the western region $(7.8 \%)$, wealthiest households $(11.5 \%)$; for children with at least high school graduate mothers $(9.8 \%)$, no siblings $(8.4 \%)$ and higher birth weights $(7.1 \%)$ Multiple logistic regression analysis, where being obese was the dependent variable, showed that the birth order and birth weight of the child and duration of breastfeeding were significant determinants of childhood obesity.

Conclusion The results of the study will be benefited by the health planners and administrators to combat this problem as early as possible.

\section{SP1-88 MORTALITY IN AN URBAN COHORT IN RAGAMA, SRI LANKA}

doi:10.1136/jech.2011.142976n.65

P V T S Vithanage, P A S Panapitiya, N Padmakumara, S Hemantha, K T A A Kasturiratne, A R Wickremasinghe, ${ }^{*}$ A Pathmeswaran, M J Pinidiyapathirage. Faculty of Medicine, University of Kelaniya, Ragama, Sri Lanka

Introduction The leading causes of mortality in Sri Lanka are due to chronic diseases. We describe the mortality patterns in a 35-64-yearold urban cohort resident in Ragama, Sri Lanka and followed over 3 years.

Methods A follow-up study was conducted among 2986 35-64 year olds randomly selected from the Ragama Medical Officer of Health 